

\section{Processus expérimentaux}

\section{Modes d'approches.}

Parmi les nombreux problèmes que nous avons pu aborder, un grand nombre était susceptible d'une étude par simulation directe. Il s'agit généralement de problèmes de transfert de chalcur ou de matière, avec ou sans effet de densité, dans lesquels la convection forcée et la diffusion turbulente jonent le ròle principal. On peut, dans ces cas, concevoir une maquette plus ou moins complète à échelle réduite permetlant une bonne reproduction de l'écoulement réel; la grandeur étudiée (température ou concentration) est matérialisée sur le modèle par une concentration en traceur dont l'évolution est directement mesurée. Les conditions aux limites sur le traceur doivent évidemment traduire celles existant réellement pour la grandeur étudiće. On concoit que dans une telle simulation directe, le traceur peut souvent ètre utilisé à la fois comme tel et comme agent provoquant l'effet de densité si la reproduction d'un tel effet est nécessaire pour la similitude des écoulements modèle et réel.

Echappent, par exemple, à une telle approche, les problèmes comportant :

a) un dégagement de puissance ou une création de malière à l'intérieur même de la masse fluide en mouvement. De nombreux cas de ce type se présentent dans les domaines nucléaires et chimiques. En toute rigueur, la simulation directe n'est pas impossible; elle présente néanmoins des difficultés de réalisation pratique;

b) une influence importante de la diffusivité moléculaire. Les problèmes thermiques concernant des milieux à très faible nombre de Prandt (tel le sodium liquide) en constituent un exemple; la simulation directe réalisée avec un fluide et un traceur courant n'est alors plus représentative de la réalité;

c) une étude détaillée ou une intervention capitale des phénomenes au voisinage des parois. Par exemple, un probleme comportant une convection libre ou mixle le long d'une paroi chauffée donne lieu à des difficultés d'ordre pratique et thérique qui le rendent inabordable par un procédé de simulation directe.

Lorsque la simulation directe n'est pas possible, on peut effectuer des expériences moins globales visant à déterminer des caractéristiques partielles qui pourront servir à remonter ensuite par un cheminement plus ou moins aisé au résultat souhaité.

Ainsi, on peut rechercher lordre de grandeur du paramètre diffusivité turbulente. Ceci est en général compliqué par le fait que toute expérience de traceur tient compte à la fois de la convection et de la diffusion et qu'il est souvent difficile de séparer les deux. On a pu néanmoins se servir de telles expériences pour caractériser les échanges thermiques au sein d'un milieu double phase défini par une phase liquide pratiquement pas débitante, a la base de laquelle le gaz ćlait injecté pour entretenir une agitation permanente. Les aspects convection et diffusion pouvaient dans ce cas être réunis dans un paramètre diffusivité globale, puisque le milieu était en moyenne immobile.
Dans le même ordre d'idées, on peut résoudre des problemes du type $a$ ) évoqué ci-dessus lorsque la puissance spécifique dégagée dans la masse est à peu près constante et pour autant que l'on n'ait pas à utiliser le traceur pour reproduire des effets de densité. On peut en effet démontrer qu'un tel problème revient à mesurer le temps de séjour moyen mis par une particule poux aller d'un point à un autre. Nous reviendrons sur ce genre de processus d'approche.

\section{Similitude.}

En l'absence de toute action importante des effets cle densité, la similitude est, bien entendu, une similitude de Reynolds complétée par l'égalité des nombres de Prandtl on de Schmidt. Dans de nombreux cas pratiques un certain écart peut être consenti sur le nombre de Reynolds, la seule condition étant que les nombres de Reynolds modele soient supérieurs à des «normes » plus ou moins bien définies, au-delà desquelles l'écoulement ne change pratiquement plus de configuration. Ces «normes » sont à déterminer sur chaque cas particulier. Leur respect n'autorise d'ailleurs pas à dire que l'éconlement modele est absolument semblable à l'écoulement réel, ce qui est toujours faux en particulier près des parois, sauf si la similitude de Reynolds est intégralement respectée. Ceci explique les difficultés d'utilisation d'une simulation directe des que les phénomènes an voisinage des parois jouent um rôle prédominant.

L'égalité des nombres de Prandtl et de Schmidt est nécessaire pour conserver des actions semblables des diffusivités moléculaires. Dans beaucoup de problèmes, ces diffusivités sont négligeables devant la diffusivité turbulente, ce qui autorise quelques libertés en ce domaine. Sur ce point, remarquons que l'utilisation de l'eau salée comme traceur fait apparaitre sur le modèle une diffusion moléculaire tout à fait négligeable.

Lorsque les forces de densité jonent un ròle important, la similitude de Fronde $(\mathscr{g}=\mathrm{V} / \sqrt{g \mathrm{D} \Delta \mathrm{p} / \mathrm{p}})$ est intéressante, car elle conduit à des modìles très aisément exploilables. Il convient néanmoins de souligner que celle similitude n'est pas a priori applicable à tous les cas. En particulier, la distorsion consentie sur le nombre de Reynolds peut joner un ròle important et conduire à des erreurs.

D'une manière générale, l'application de ces différentes règles de similitude est toujours basée sur Ie fait que les phénomènes de difusion (de quantité de mouvement, de matière ou de chaleur) à l'échelle moléculaire sont pratiquement négligeables devant les phénomenes de difusion turbulente et que ceux-ci deviennent pratiquement indépendants des premiers dès que les nombres de Reynolds sont assez grands. Le caractère hautement utilitaire d'une telle proposition ne doit pas faire oublier qu'elle n'est sourent qu'une approximation dont le domaine de validité demande à etre regardé dans chaque cas particulier.

\section{Instrumentation.}

Pour mener à bonne fin les essais selon les méthodes décrites plus haut, nous avons dù mettre an point un appareillage nouveau. 


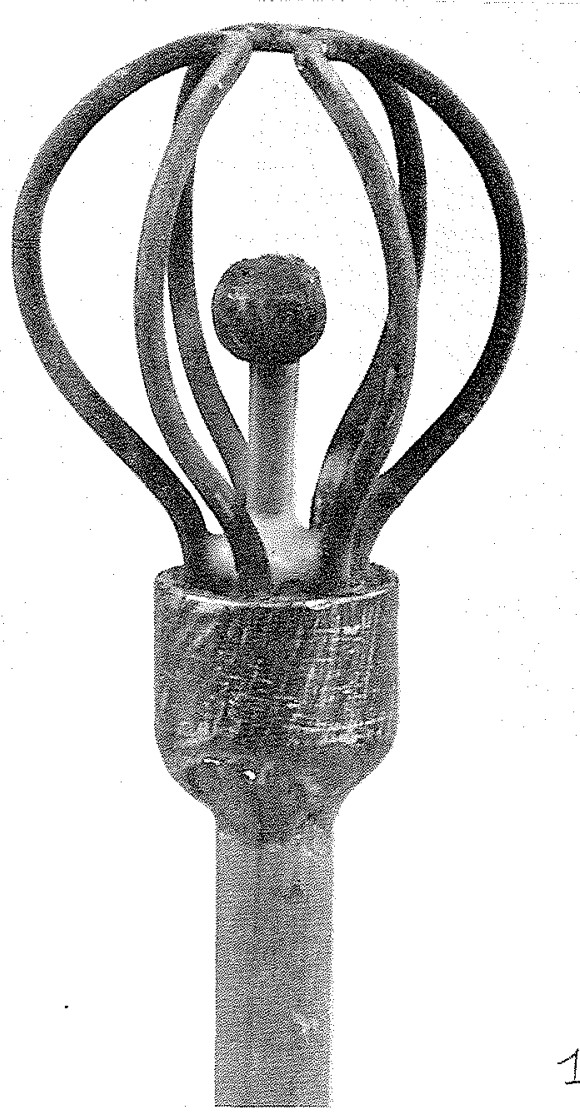

1/ Sonde / probe.

Le mesure de la concentration saline d'une solulion se ramène à celle de sa conductivité, qui, pour de faibles concentrations, est une fonction pratiquement linéaire de la teneur en sel. Cette mesure se ramène donc à celle de la conductance d'une sonde de coefficient géométrique connu.

Une solution saline élant un milieu électrolytique, pour que les mesures soient stables et reproductibles, il est nécessaire de choisir :

- la fréquence d'alimentation de la sonde pour avoir une bonne caractéristique d'impédance;

- le métal pour limiter les effets de polarisation de la surface des électrodes;

une valeur suffisamment faible du gradient de tension au voisinage des électrodes pour éviter la polarisation.

Nous nous sommes orientés vers des fréquences d'alimentation de $2000 \mathrm{~Hz}$, des tensions de 0,3 à $0,5 \mathrm{~V}$ el des électrodes en nickel sablé.

Ia sonde (photo) est constituce de deux électrodes concentriques, ce qui supprime les effets de bords. L'électrode centrale est une sphère pleine de $1 \mathrm{~mm}$ de diametre environ et l'électrode extérieure est composée de 4 on 6 anneaux de 6 à $10 \mathrm{~mm}$ de diamètre, ce qui rend la sonde omnidirectionnelle.

Le coefficient géométrique de la sonde ne dépend pratiquement que du diamètre de la sphère intérieure, qui, étant petite, rend la sonde pratiquement ponctuelle.

Le temps de réponse d'une telle sonde est de l'ordre de $0,1 \mathrm{~s}(0,3 \mathrm{~s}$ dans les plus mauvaises conditions).
La chaine comprend une alimentation à tension constante et un dispositif électronique de détection du courant traversant la sonde. Un amplificateur délivrant une tension proportionnelle à ce courant, donc à la concentration de la solution, permet d'attaquer un enregistreur ou un organe de calcul.

\section{Mélange de deux jețs en régime permanent}

\section{Problème posé.}

Le problème nous a été posé par la région d'Equipement nucléaire III de l'E.D.F., dans le cadre des études de la détection de ruplure de gaine (DRG) du réacteur Bugey 1.

Les éléments combustibles de Bugey 1 sont du type annulaire, avec circulation de $\mathrm{CO}_{2}$ dans l'espace circulaire au centre de l'élément et dans l'espace annulaire entre l'élément et le canal.

Si la gaine entourant le produit combustible se rompt, les produits de fission vont contaminer le $\mathrm{CO}_{2}$ et l'ensemble du circuit.

La détection de rupture de gaine s'effectue en prélevant du $\mathrm{CO}_{2}$ à l'aval de chaque canal et en analysant ses propriétés radioactives.

Il s'agissait pour nous d'étudier le mélange des deux débits, débit central et débit annulaire, pour définir la position optimale du point de prélèvement afin que le gaz prélevé soit représentatif de la pollution moyenne de l'ensemble du débit.

\section{Similitude et échelle.}

Pour accroître les échanges thermiques entre l'élément et le gaz, la surface extérieure de la gaine comporte des chevrons d'environ $0,3 \mathrm{~mm}$ d'épaisseur, de $8 \mathrm{~mm}$ de hauteur, espacés de $1,8 \mathrm{~mm}$ et faisant un angle de $20^{\circ}$ avec une génératrice.

Un tel état de surface devant être un facteur d'homogénéisation, nous avons effectué les essais avec des gaines réelles, donc à l'échelle 1.

Pour nous affranchir d'essais au gaz, en pression el en température - toujours très lourds et coûteux - nous avons utilisé de l'eau avec un débit global de $18 \mathrm{l} / \mathrm{s}$, ce qui nous a conduit à une distorsion du nombre de Reynolds $\mathcal{R}$ de l'ordre de 13 à 26, les nombres de Reynolds du modèle restant toutefois supérieurs à $5,5 \cdot 10^{4}$.

\section{Modèle.}

Les études furent effectuées sur une section d'essais représentant (fig. 2.) :

- la partie supérieure du canal sur une hauteur de $1250 \mathrm{~mm}$ environ;

- la partie aval du canal, schématisée par une manchelte de $180 \mathrm{~mm}$ de diamètre et $1500 \mathrm{~mm}$ de longueur. En fait, l'étude définitive fut effectuée sur une géométrie beaucoup plus complexe, mais dont le particularisme n'offre aucun intérêt ici.

\section{Processus expérimental.}

La méthode consistait à déterminer la concentration locale $\mathrm{C}_{1}$ en chlorure de sodium dans l'eau après injection d'une solution mère et à la compa- 
rer à la concentration $\mathrm{C}_{0}$ obtenue après homogénéisation totale.

Cette solution-mère était injectée parallèlement à l'écoulement et à la même vitesse que lui.

La concentration locale était mesuréc à l'aide des conductimètres décrits précédemment.

\section{Résultats.}

Nous n'indiquerons rapidement ici que les résultats obtenus sur la géométrie la moins parliculière étudiée - celle où l'aval du canal est une manchette cylindrique.

Les conditions expérimentales sont:

- essais à l'eau;

- vitesse dans l'espace central...... $1,7 \mathrm{~m} / \mathrm{s}$,

- vitesse dans l'espace annulaire.... 1,15 m/s,

- vitesse moyenne en pleine section. . $0,7 \mathrm{~m} / \mathrm{s}$.

L'homogénéisation fut étudiée dans quatre plans situés à 900, 1200, 1500 et $1800 \mathrm{~mm}$ du bas du dernier élément, selon deux diamètres perpendiculaires.

Trois types de pollution furent étudiés :

1. Pollution homogène du canal central (fig. 3) conduisant à une bonne symétrie et à une homogénéisation relativement bonne;

2. Pollution localisée à la paroi du canal central (fig. 4). On constate une dissymétrie et une homogénéisation systématiquement moins bonne;

3. Pollution localisce à la paroi interne du canal périphérique (fig. 5). On constate une dissymétrie très marquée et une homogénéisation s'accroissant rapidement jusqu'à $1200 \mathrm{~mm}$ et évoluant plus lentement après.

Ces quelques essais nous montrent que l'homogénéisation croìt rapidement dans les 1200 premiers millimètres et évolue lentement après, ce qui nous a conduits à placer les prises de prélèvement de la DRG à $1200 \mathrm{~mm}$ environ.

Cette série d'essais préliminaires sur une géométrie simple fut suivie d'essais sur la géométrie réelle, beaucoup plus complexe, selon une méthode identicue.

\section{Propagation d'un front raide de température engendrant des effets de densité}

\section{Problème posé.}

Cette étude a été faite à la demande du service d'étude mécanique et thermique des réacteurs rapides du CEA, dans le cadre des études d'avant-projet de la cuve primaire du réacteur Phénix.

Le réacteur Phénix est du type rapide, refroidi au sodium, d'une puissance thermique de $600 \mathrm{MW}$ (fig. 6). Le sodium issu des assemblages à $560^{\circ} \mathrm{C}$ pénètre dans une cuve à surface libre. Il repasse ensuite dans la cuve principale à travers six échangeurs, où il est refroidi de 560 à $400^{\circ} \mathrm{C}$. Il est repris dans cette cuve principale par trois pompes et renvoyé dans les assemblages où il est réchauffé de 400 à $560{ }^{\circ} \mathrm{C}$.
En cas d'incident provoquant une chute de barre, la puissance du réacteur décroìt brusquement à une valeur très faible et la température du sodium à la sortie des assemblages passe de 560 à $400^{\circ} \mathrm{C}$ en $5 \mathrm{~s}$ environ; du sodium à $400^{\circ} \mathrm{C}$ pénètre dans la cuve encore à $560^{\circ} \mathrm{C}$, d'où des risques de contraintes inacceptables pour les structures. Il importe donc de comnaitre l'atténuation par diffusion qu'a subie le front froid issu du coeur au moment où il se présente au voisinage des parois, compte tenu de l'écoulement non isotherme qu'il occasionne.

\section{Similitude et échelle.}

Nous disposions pour cela d'une maquette à l'échelle $1 / 4$, à l'eau, dans laquelle nous avons injecté de l'eau salée simulant le sodium froid.

Cette injection d'eau salée était destinée, d'une part, à servir de traceur et, d'autre part, à représenter les différences de densité entre sodium chaud et sodium froid selon la similitude de Froude.

Cette analogie, transfert de chaleur - transfert le matière, n'est pas parfaitement admissible ici, car dans le sodium la conduction est un facteur d'échange très important qui, considéré seul, peut produire des effets du mème ordre de grandeur que ceux engendrés par la diffusion turbulente.

Ne pas simuler cette conduction, conduit a observer sur le modèle des atténuations plus faibles que dans la réalité et par suite à exalter les effets de densité.

Une telle distorsion est admissible dans la mesure où, d'une part, les effets de densité ont peu d'influence (les essais l'ont confirmé) el où, d'autre part, les résultats auxquels elle conduira seront pessimistes par rapport à la réalité et iront donc dans le sens d'une sécurité accrue.

Les échelles - grandeur modèle sur grandeur réelle - étaient :

$$
\begin{aligned}
& \text { - géométrie. ........ } 1 / 4 \\
& \text { _- vitesse. . . . . . . . . 1/2 } \\
& - \text { temps........... 1/2 } \\
& \text { - densité. ........... } 1
\end{aligned}
$$

Par l'intermédiaire des densités, nous avions une corrélation entre la température et la concentration en sel (l'écart de densité entre 560 et 400 était obtenu par une concentration de $70 \mathrm{~g} / 1$ environ).

\section{Processus expérimenial.}

On injectait à l'amonl du coeur une solution saline dont le débit el la concentration étaient calculés pour reproduire à la sortie du cour l'évolution de densité souhaitée.

L'évolution des concentrations dans la cuve était suivie en différents points à l'aide des conductimètres décrits prócédemment.

\section{Résultats.}

Les essais, ont permis d'obtenir les coefficients d'atténuation cherchés (fig. 7). De plus, il ont montré que, pour des débits cour modèle variant de 1 à 2 ou des gradients variant de 1 à 10 , les résultats étaient pratiquement identiques, c'est-à-dire que la convection forcée était nettement plus importante que la convection libre. 

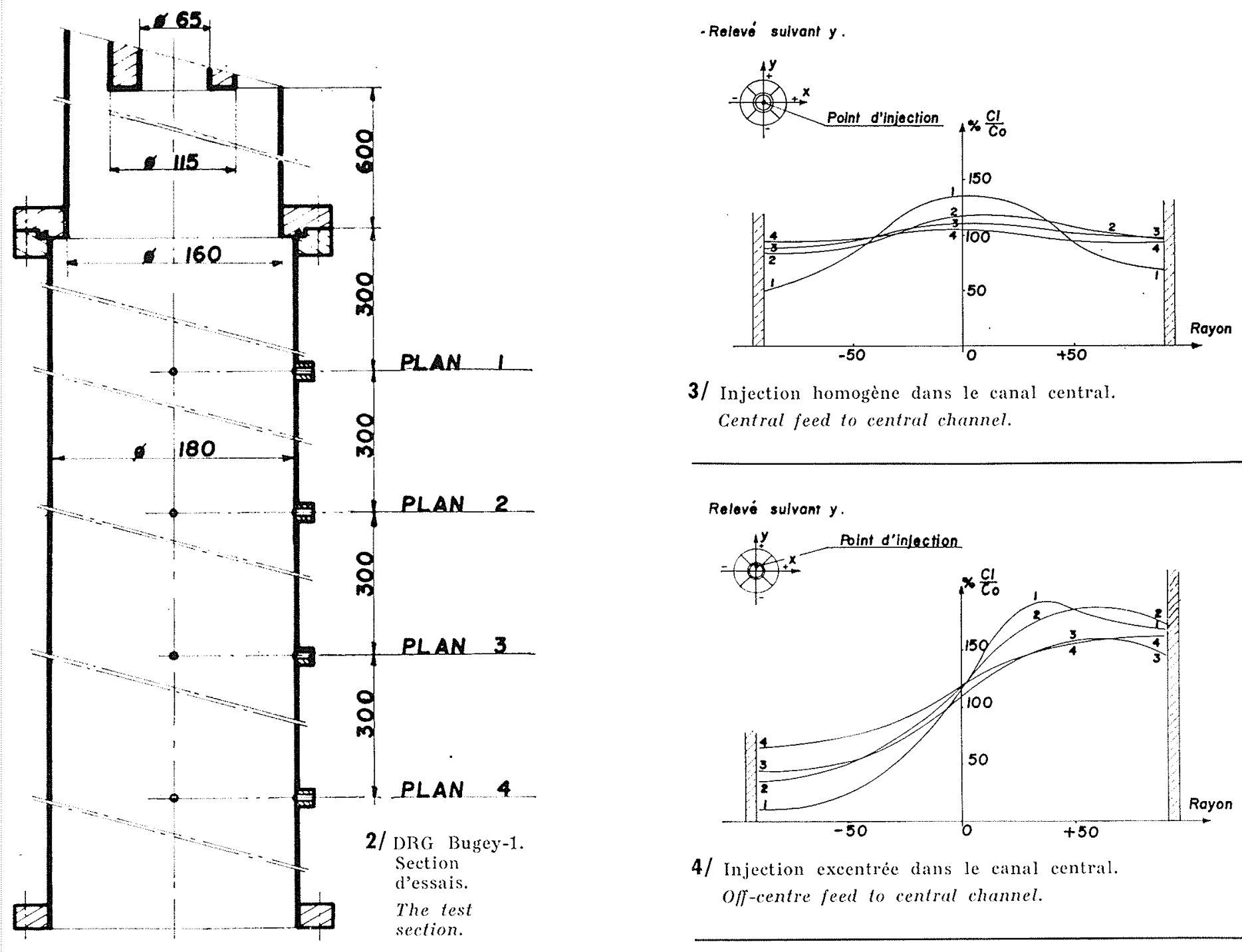

3/ Injection homogène dans le canal central. Central feed to central channel.

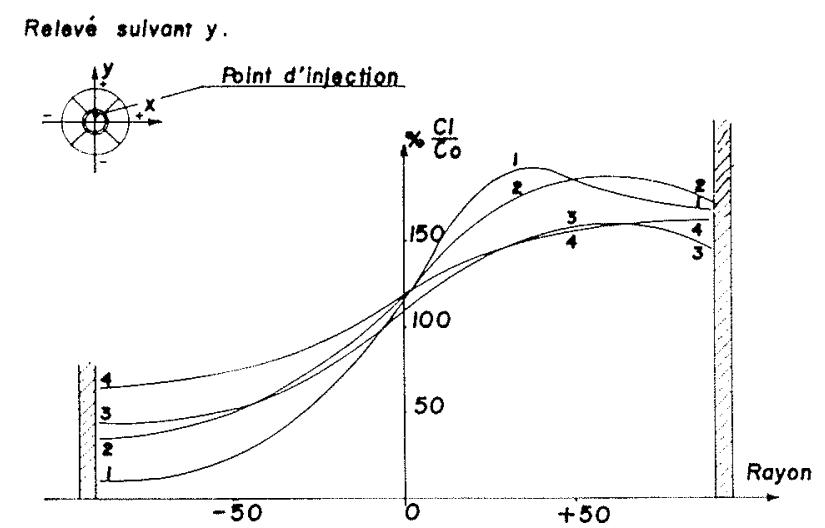

4/ Injection excentrée dans le canal central. off-centre feed to central channel.
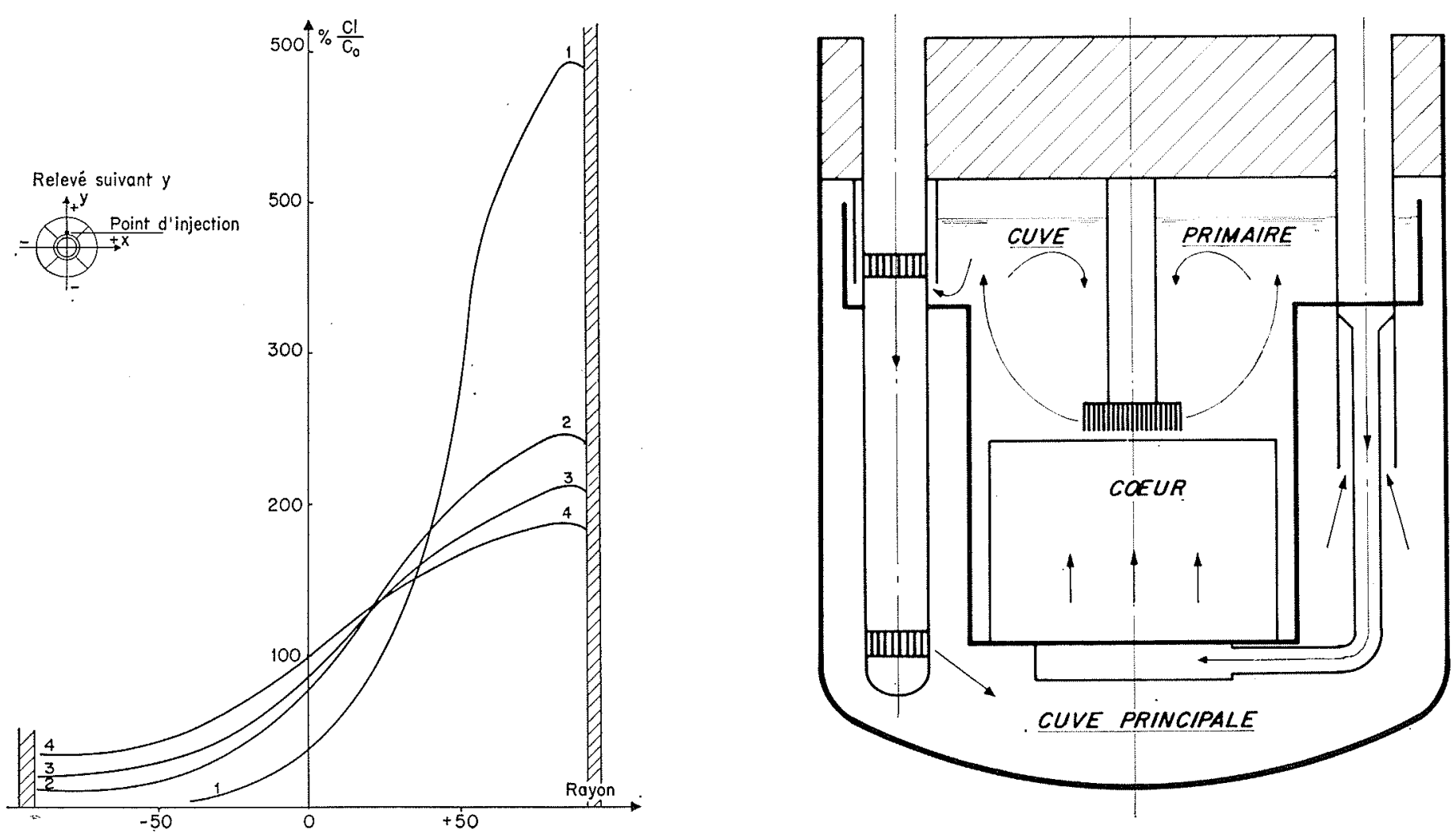

5/ Injection excentrée dans le canal annulaire. off-centre feed to annular channel.

6/ Réacteur Phénix. Schéma de principe du circuit primaire. Phénix reactor. Primary circuit. 


\section{Détermination \\ des temps de séjour dans un réacteur homogène}

\section{Problème posé.}

Le probleme que nous abordons maintenant a déjà été présenté un peu différemment au cours d'un Comité Technique de la Société Hydrotechnique de France sur les courants de densité (*). Résumons rapidement les aspects qui nous intéressent ici.

On désire organiser un écoulement d'eau dans un récipient de forme plus ou moins sphérique (dans le cas particulier objet de notre précédente publication, cette eau véhicule une suspension contenant de l'uranium qui donne lieu à une réaction nucléaire de fission dès que la masse crilique est atteinte). Le débit pénètre et sort de ce récipient respectivement à sa base et à sa partie supéricure par l'intermédiaire d'une tuyauterie dont le diamètre représente environ le $1 / 5^{*}$ du diamètre de la sphère.

L'écoulement doit satisfaire à deux criteres principaux :

a) les vitesses au voisinage de la paroi inférieure du récipient doivent être assez fortes pour prévenir tout dépòt de produit en suspension dans l'eau;

b) le mélange dans le récipient doit être suffisamment intense pour que le dégagement de puissance par unité de volume à peu près uniforme qui s'effectue au sein de fluide présent dans le récipient, ne domne pas naissance à des surtempératures locales exagérées (à la limite, on concoit que la qualité du mélange est aussi utile pour conserver une bonne homogénéité des concentrations de produit en suspension).

Un tel problème se pose effectivement pour le cour des réacteurs nucléaires homogènes, mais sous des formes assez voisines, on peut le retrouver dans le domaine du génie chimique.

\section{Elude expérimentale.}

Pour satisfaire au premier critèe énoncé plus haut, on peut faire appel à un distributeur d'entrée alimentant par priorité les zones voisines de la paroi. Une légère mise en rotation du fluide facilite les choses et nous verrons d'ailleurs plus loin que cette dernière idée peut être exploitée á fond pour résoudre le probleme en provoquant une centrifugation importante de l'écoulement.

Le distributeur retenu donne un écoulement comportant une vaste zone de recirculation centrale plus ou moins instable décrite dans la figure 8.

L'étude du critère $b$ ) ne peut pas, comme nous l'avons dit en commencant, etre basée sur une simulation directe, étant donné les difficultés de réalisation pratique auxquelles conduit la mise en ceuvre d'une puissance dégagée dans la masse. En fait, le problème de la détermination du champ des températures dans le récipient se ramène à la connaissance du temps que met, depuis l'entrée, me particule fluide statistique moyenne pour parvenir en

(") La Houille Blanche, n" 1/1965. un point donné. C'est, en effet, durant ce temps que cette particule doit absorber une puissance constante. L'échauffement qu'elle subit détermine Ia température atleinte au point considéré.

\section{Appelons :}

$p$ el $c$ les masse et chaleur spécifiques du fluide;

$\mathrm{P}$ la puissance dégagée dans la masse par unité de volume;

Q le débit;

$V$ le volume total du récipient dans lequel se dégage une puissance totale $\mathscr{P}=\mathrm{PV}$;

$\tau$ le temps mis par la particule statistique moyenne pour aller de l'entrée à un point quelconque $M$ du récipient $\left(\tau_{M}\right)$ ou à la section de sortie $\left(\tau_{*}\right)$;

$\mathrm{T}$ la température, à l'entrée $\left(\mathrm{T}_{3}\right)$, au point $\mathrm{M}\left(\mathrm{T}_{\mathrm{M}}\right)$, à la sortie $\left(\mathrm{T}_{*}\right)$.

On a :

$$
\mathrm{T}_{\mathrm{M}}-\mathrm{T}_{\mathrm{P}}=\frac{\mathrm{P}}{\rho \mathrm{c}} \tau_{\mathrm{M}}
$$

et en particulier :

d'où :

$$
\mathrm{T}_{s}-\mathrm{T}_{\mathrm{s}}=\frac{\mathrm{P}}{\rho c} \tau_{s}=\frac{\mathrm{PV}}{\rho c \mathrm{Q}}=\frac{\mathscr{P}}{\rho c \mathrm{Q}}
$$

$$
\mathrm{T}_{\mathrm{M}}-\mathrm{T}_{\mathrm{s}}=\mathrm{A}\left(\mathrm{T}_{\mathrm{s}}-\mathrm{T}_{\mathrm{s}}\right)
$$

A est l'âge relatil au point $\mathrm{M}$ qui est égal par définition à $\tau_{\mathrm{M}} / \tau_{\mathrm{s}}$.

Les mesures ont été effectuées sur un modèle échelle 1 du réacteur alimenté en eau avec le débit réel.

Dans ces conditions, les temps $\tau$ à mesurer varient entre 0,5 et $3 \mathrm{~s}$. Une faible distorsion du nombre de Reynolds est occasionnce par le fait que le réacteur réel représenté fonctionne à haute température, d'où une différence de viscosité sensible. L'eau salée est utilisée comme traceur pour déterminer $\tau$ en chaque point par la méthode suivante:

$A$ l'instant $t=0$, un step de concentration d'amplitude $C_{0}$ est produit à l'entrée du récipient et l'on mesure la concentration $\mathrm{C}_{\mathrm{M}}(t)$ qui en résulte au point considéré M. Le step à l'entrée étant obligatoirement imparfait (on passe de $\mathrm{C}_{\mathrm{E}}=0$ à $\mathrm{C}_{\mathrm{E}}=\mathrm{C}_{0}$ en $0,2 \mathrm{~s}$ environ), on mesure simulanément $\mathrm{C}_{\mathrm{x}}(t)$ dans la section d'entrée. On a alor's :

$$
\tau_{\mathrm{MI}}=\int_{0}^{\infty} \frac{\mathrm{C}_{\mathrm{I}}(t)-\mathrm{C}_{\mathrm{M}}(t)}{\mathrm{C}_{0}} d t
$$

Les signaux délivrés par les deux sondes placées à l'entrée et au point $M$ sont traités électriquement. Apres une correction tenant compte de la non-identité des caractéristiques des deux sondes utilisées, on élabore la différence des deux grandeurs, puis l'intégrale de cette différence dans le temps. Le résultat est lu directement sur un microampèremètre (fig. 9). La limite supérieure de l’intégrale est ćvidemment finie et on réalise une seconde mesure identique pour le step négatif qui ramène $\mathrm{C}_{\mathrm{D}}(t)$ de $\mathrm{C}_{0}$ à 0 . Les deux résultats sont en général voisins, sauf dans des zones proches de l'entrée où l'instabilité de l'écoulement engendre une légère dispersion.

La valeur de $\mathrm{C}_{0}$ utilisée est de $5 \mathrm{~g} / \mathrm{l}$ environ. Cette valeur n'occasionne pas de perturbation sensible lice à l'apparition d'éventuels courants de densité au cours du transitoire. Le problème des courants 


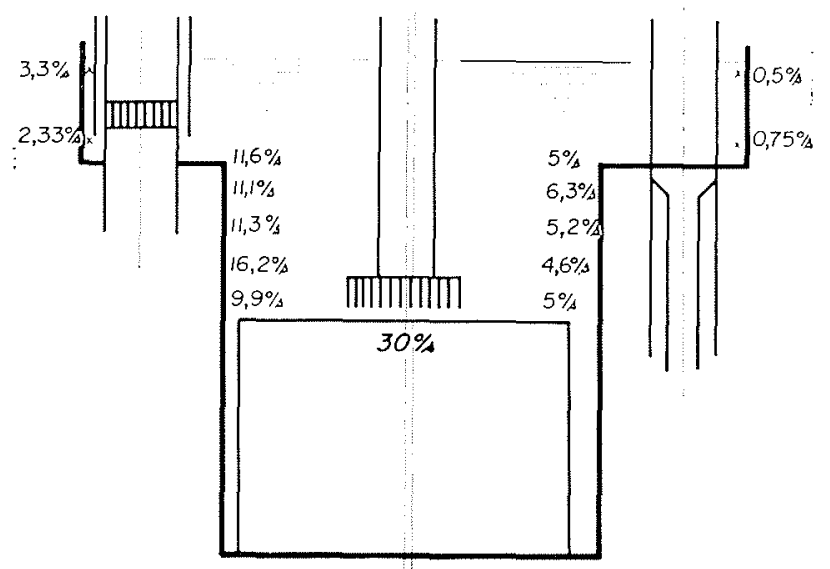

7/ Réacteur Pliénix. Atténuation d'un choc froid. Phénix reactor. Cold front attenuation.

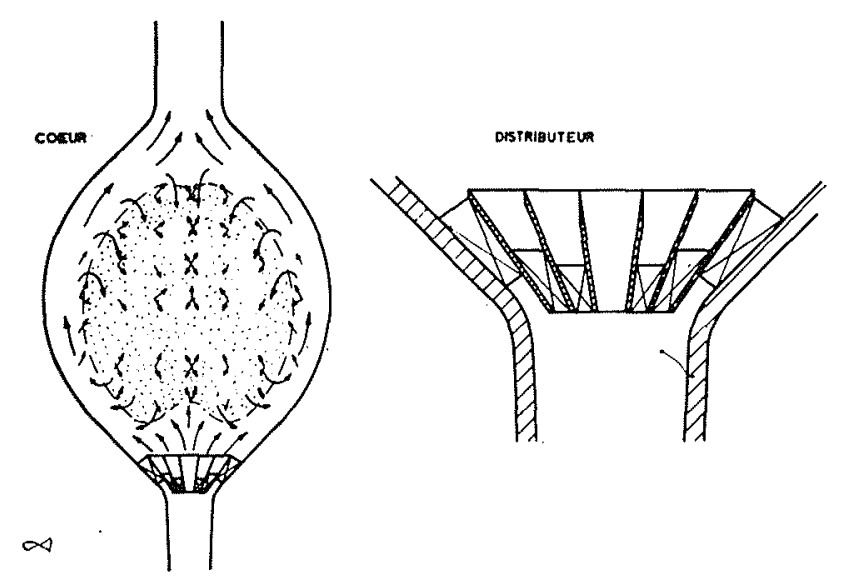

8/ Réacteur homogène. Distributeur et écoulement observé. Homogeneous reactor. Distributor and observed flow.

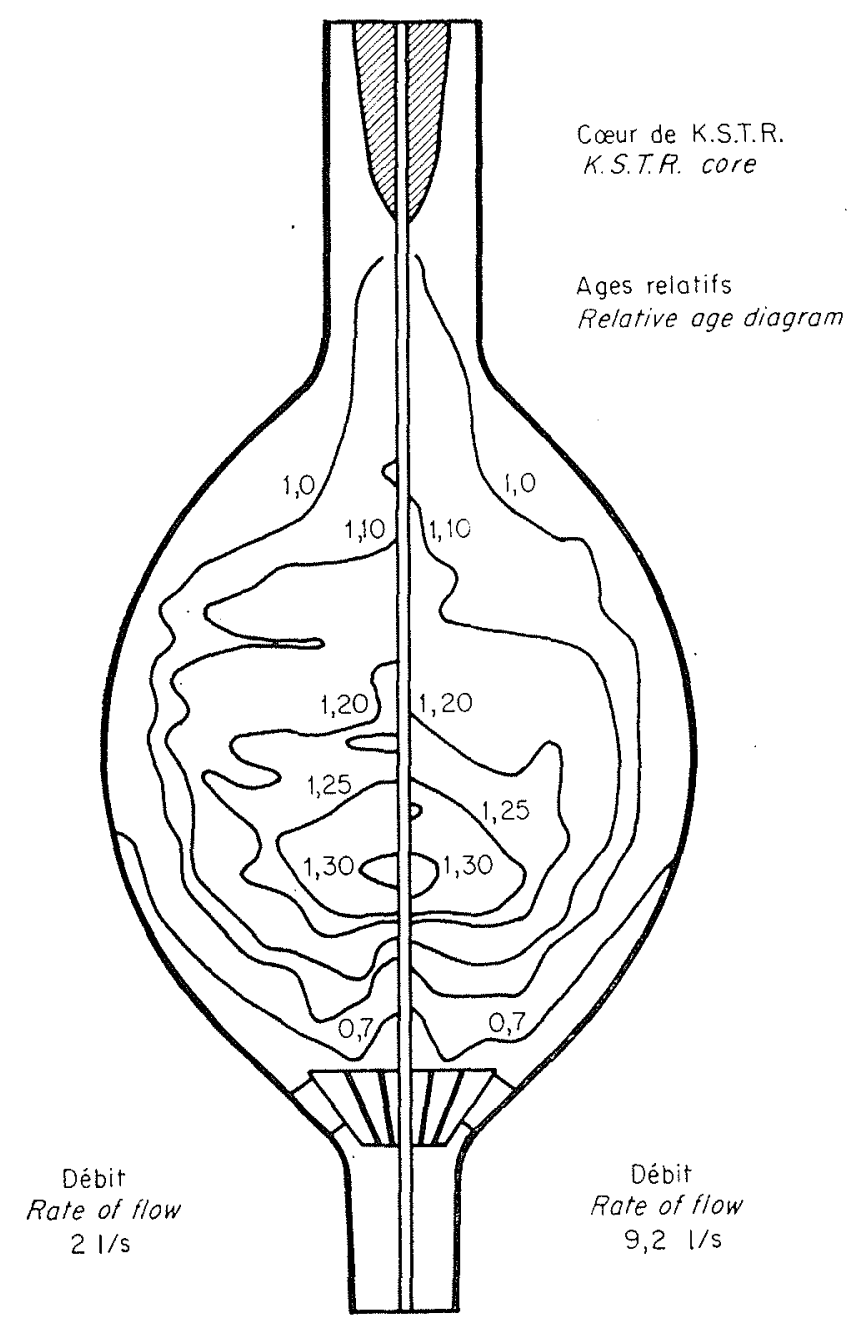

10/ Réacteur homogène. Résultats expérimentaux. Homogeneous reactor. Experimental results.

I/ Réacteur homogène.

Dispositif expérimental.

Homogeneous reactor. The experimental rig.

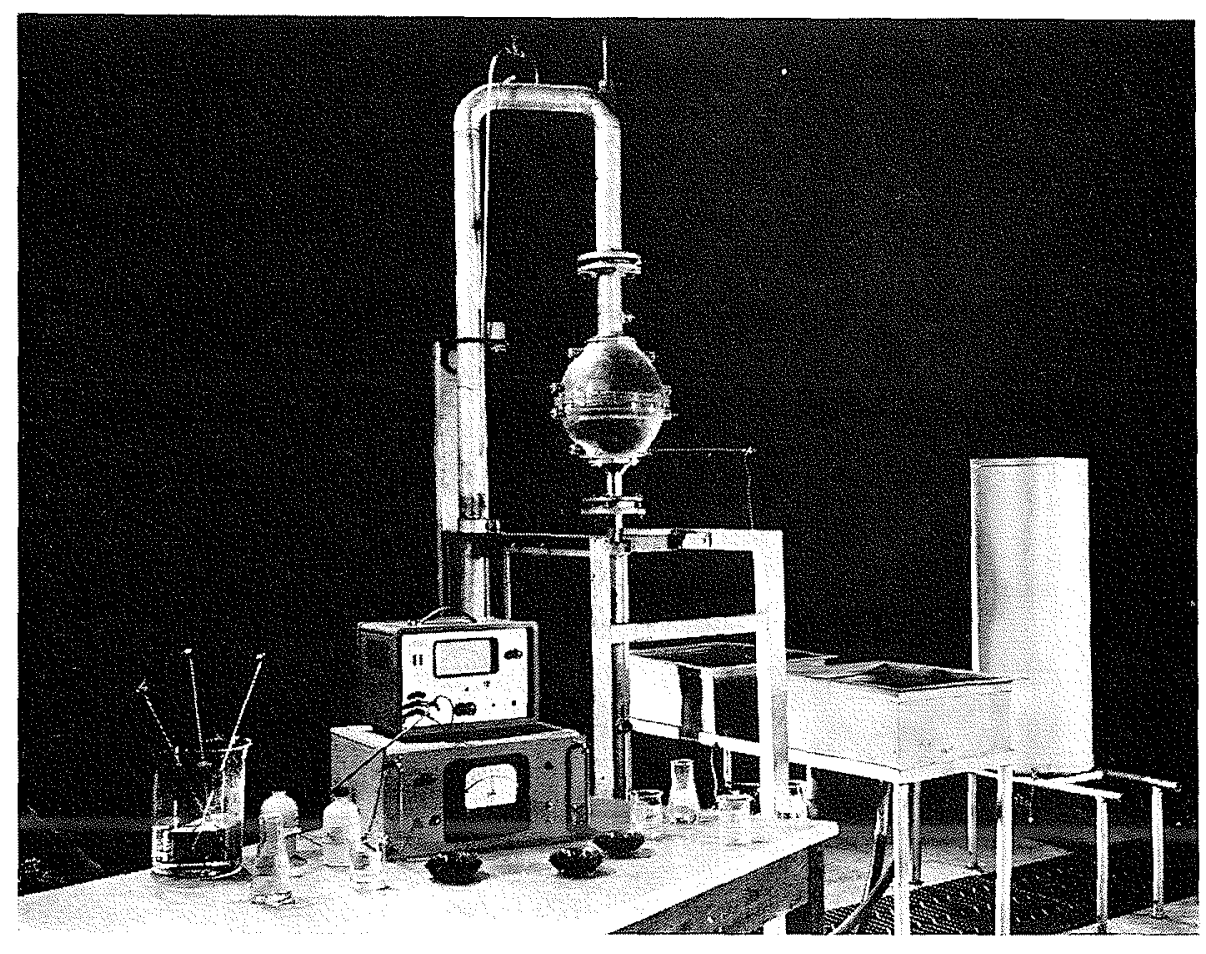




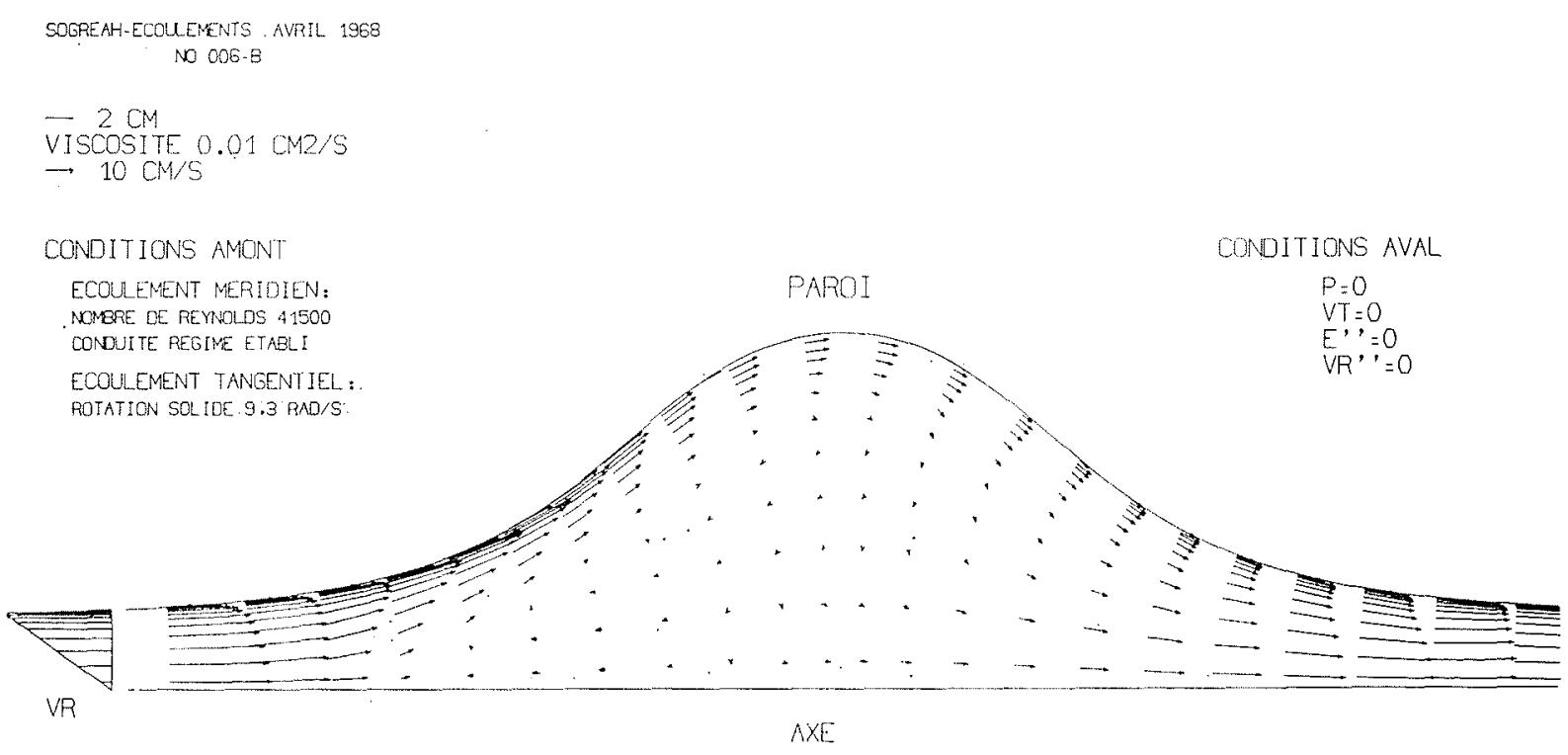

11/ Programme Pétula. Divergent, convergent de révolution - champ de vitesses calcule.

Pétula programme. Velocity pattern in an expanded duct section.

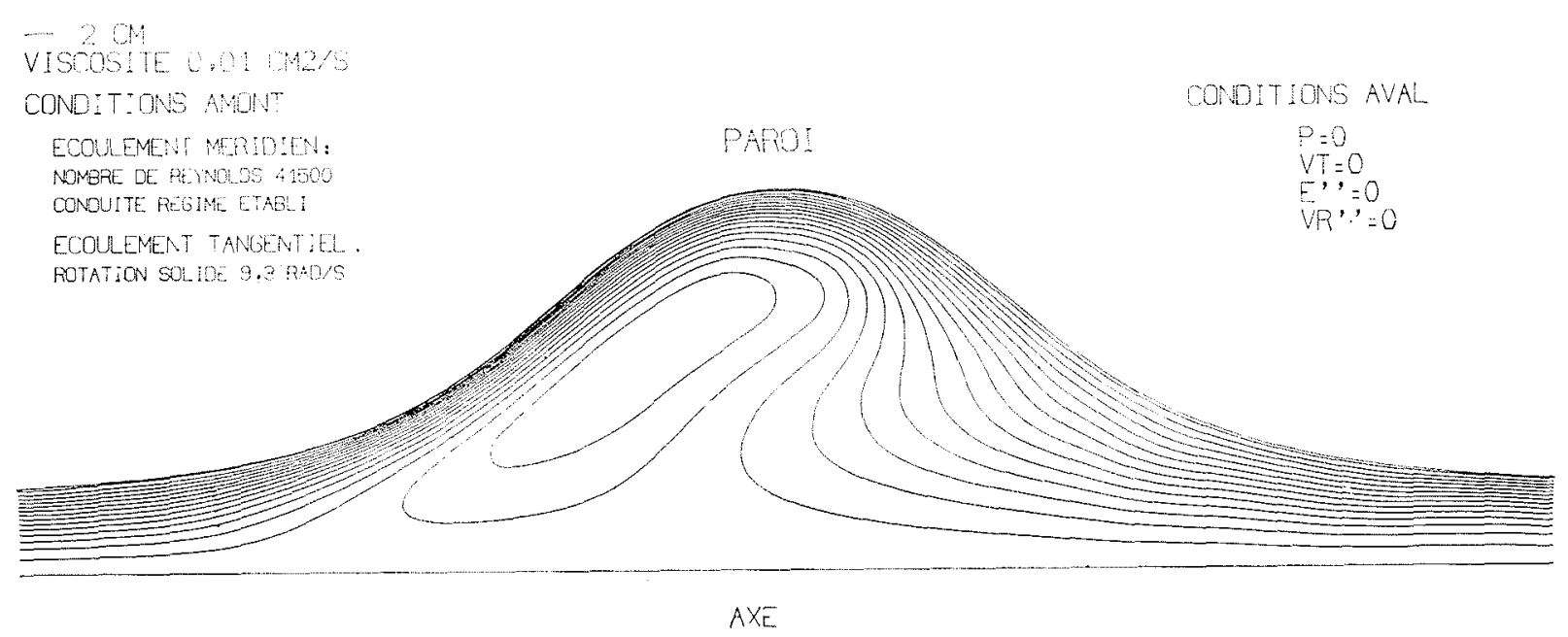

12/ Programme Pétula. Divergent, convergent de révolution -- lignes de courant calculées.

Pétnla programme. Streamlines in an expanded duct section.

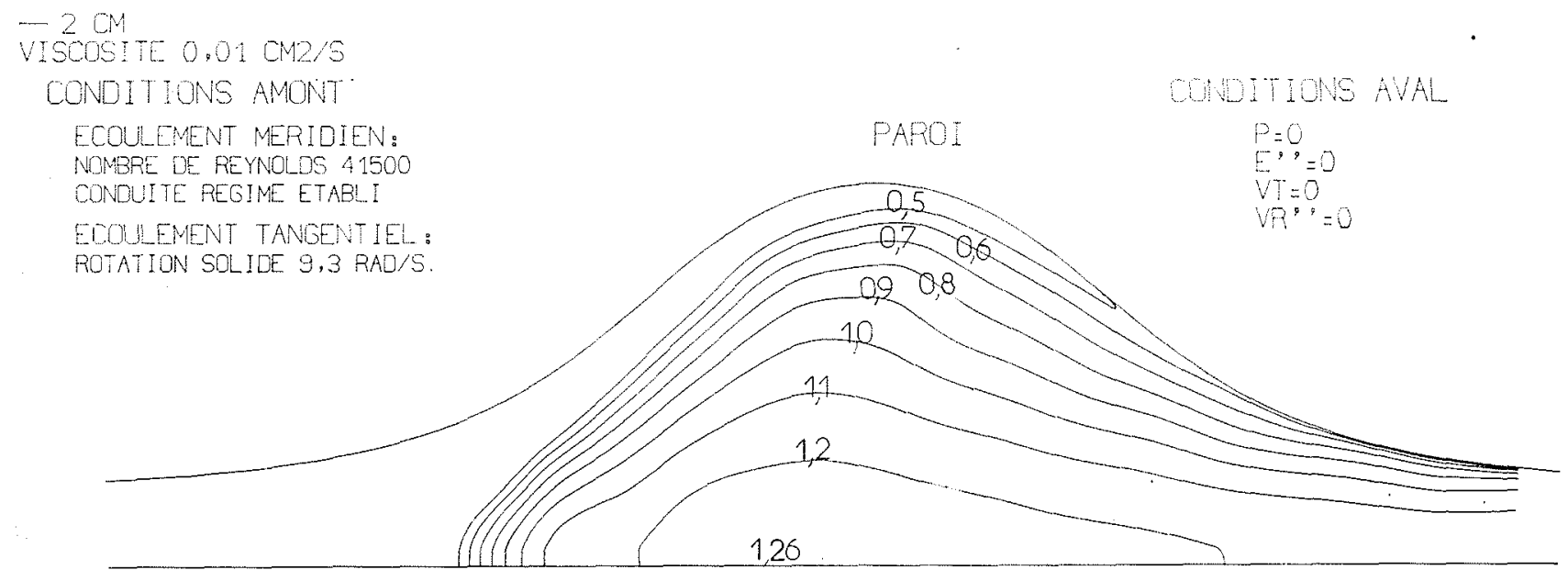

AX'E

13/ Divergent-convergent de révolution. Ages relatifs / Relative age pattern in an expanded duct section. 
de densité en permanent sur le réacleur faisait l'objet de la première publication. Nous n'y reviendrons pas, disons simplement qu'on a pu arriver à la conclusion que ces courants étaient vaisemblablement négligeables.

La figure 10 donne une moyenne de l'ensemble des résultats obtenus. Ceux-ci concernent environ 3000 essais du type ci-clessus pour des débits variant de 2 à $9 \mathrm{l} / \mathrm{s}$, c'est-à-dire pour des nombres de Reynolds à l'entrée variant de 50000 à 300000 . On peut noter que la carte des âges relatifs semble relativement insensible à cette valeur de Reynolds dans la gamme explorée, ce qui permet d'extrapoler sans peine au nombre de Reynolds réel qui est un peu supérieur.

\section{Etude théorique.}

Le distributeur mis au point dans l'étude que nous venons de décrire présente quelques inconvénients pratiques; il est relativement délicat à construire, et l'on peut craindre que l'érosion par une suspension très dure n'en change les caractéristiques assez rapidement. On a donc pensé utiliser uniquement les propriétés des écoulements en rotation pour produire des vitesses importantes à la paroi, en particulier dans la partie divergente du récipient.

Quelques expériences ont ainsi été faites dans ce sens : une rotation solide est créée à l'entrée par une vrille hélicoïde comportant suffisamment de canaux élémentaires pour que le fluide soit correctement mis en rotation sur une distance pas trop importante. Les résultats, du point de vue vitesse, ont montré que le critère $(a)$ pouvait assez bien être rempli. Les mesures concernant le critère (b) n'ont pas encore été effectuées.

Néanmoins, dans un premier stade, on a pu tenter une application sur ce cas particulier des programmes généraux de calculs d'écoulements en cours de mise au point à Sogréah.

Nous ne nous étendrons pas sur ces programmes, objets d'articles actuellement en cours de parution. Très brièvement, ces programmes PETula (programmes d'écoulement laminaires et turbulents) traitent les équations de Navier-Stokes dans une géométrie donnée à deux dimensions ou de révolution. La turbulence (s'il y a lieu) y est simulée par des formulations semi-empiriques qui ont été adaptées pour rendre compte de lois fondamentales connues et bien vérifiées par l'expérience. Bien que tout ne soit pas encore parfait dans ce domaine, le champ d'application semble, à l'heure actuelle, suffisamment large pour que l'on puisse faire appel à de tels calculs pour obtenir des éléments d'information qui guident les expériences ultérieures.

Les figures 11 et 12 montrent le champ de vitesses et les lignes de courant obtenus par le calcul pour un convergent-divergent alimenté par un écoulement d'eau en conduite lisse en régime établi (dont le nombre de Reynolds est de 41500 ), auquel on a superposé une composante tangentielle $\omega r$. La valeur de $\omega$ est de $6,2 \mathrm{rad} / \mathrm{s}$, ce qui amène une vitesse maximale de rotation près de la paroi, dont l'ordre de grandeur est très voisin de celui de la vitesse méridienne sur l'axe.

La figure 13 donne les courbes iso-âge relatif, déterminées toujours par le calcul, en résolvant l'équation:

$$
\mathrm{V} \operatorname{grad} \mathrm{T}=\operatorname{div} \alpha \operatorname{grad} \mathrm{T}+\mathrm{P} / \rho c
$$

qui fournit l'équilibre des températures 'T en régime permanent, compte tenu de la puissance $\mathrm{P}$ dégagée par unité de volume;

$\mathrm{V}$ est le vecteur vilesse local dans le plan méridien;

$\alpha$ est la diffusivité apparente locale de chaleur, calculée en considérant a priori un nombre de Prandtl turbulent de 1.

On impose dans le secteur d'entrée une température uniforme $\mathrm{T}=\mathrm{T}_{0}$. On obtient dans le secteur de sortie des températures dont la moyenne débitante est $\mathrm{T}_{s}$. Par suite, on peut calculer en tout point :

$$
\mathrm{A}=\frac{\mathrm{T}-\mathrm{T}_{0}}{\mathrm{~T}_{\mathrm{s}}-\mathrm{T}_{0}}
$$

On constate que les valeurs de A semblent étayer la validité de la solution étudiée, compte tenu du fait que la géométrie du divergent-convergent calculé est un peu moin sévère que celle qui a fait l'objet des essais ci-dessus. Il convient néanmoins de compléter ces calculs par une étude expérimentale du même type que la précédente, car si les particularités du champ de vitesse mises en évidence par le calcul :

- recirculation axiale au début de la divergence;

- tendance dans la convergence à un rassemblement des lignes de courant sur l'axe avec une vitesse angulaire de rotation très élevée;

ont pu être vérifiées par certaines expériences préliminaires, nous ne savons pas encore si les valeurs de diffusivités turbulentes issues du calcul sont absolument conformes à la réalité. Dans certaines zones, le champ des vitesses est en effet peu sensible à ces valeurs, si bien qu'il ne suffit pas de reproduire assez bien celui-ci pour être certain des valeurs de diffusivités qui ont servi à le calculer.

\section{Conclusion}

Les quelques exemples présentés montrent que, dans les problèmes industriels, le rôle de la diffusion turbulente est souvent très important et qu'une analyse correcte des phénomènes implique des études incluant les effets qu'elle engendre. Néanmoins, on est souvent amené à une vision globale convection-diffusion qui seule peut être atteinte par des expériences simples et directement exploitables.

L'utilisation de l'eau salée comme traceur sur les modèles réduits à l'eau permet aujourd'hui d'aborder avec une grande souplesse ces expériences, même dans le cas où les écoulements étudiés sont particulièrement compliqués et où des évolutions transitoires très rapides doivent être mises en ouvre et mesurées. Par ailleurs, elle permet la reproduction d'effets de densité qui, souvent, accompagnent les autres phénomènes dans ce genre de problème.

Certaines approches directes par le calcul ont été évoquées; il y a là une voie nouvelle qui demande à être développée. Elle se heurte à de nombreuses difficultés de toutes sortes dont la moindre n'est pas la complexité physique des phénomènes à simuler. 


\section{Discussion}

En ouvrant la séance, M. le Président donne la parole a M. Hurpexus pour la présentation de la communication qu'il a rédigée en collaboration avec MM. Geneste et LAMBERT.

M. le Président remercie vivement M. Hurfenus et ouve la discussion.

Quelles sont les rajsons qui rous ont fait adopter une forme de sonde sphérique ? Tne sonde constitue par deux electrodes simples peut-elle convenir? demande M. Grus.

M. HuFresus répond que la sonde utilisée matérialisant les méridiens d'une petite sphère présente l'avantage d'être omnidirectionnelle et de mesurer la concentration dans un domaine geométriquement bien défini; en outre, elle est très robuste et son italonnage -- en conductivité - est quasi invariable. Rien ne s'oppose, en principe, a l'emploi d'une sonde plus simple, constituée, par exemple, par deux électrodes filiformes, encore que l'on puisse craindre des variations de géometrie et des effets de bords importants; il faut éviter les électrodes comportant des surfaces planes, car elles ne sont pas omnidirectionnelles.

M. Povx demande :

" $1^{\circ}$ Quelle est la concentration $C$ maximale utilise lors de l'injection d'un échelon de concentration a l'entrée du modèle sphérique?

«2० Le temps de réponse annoné pour la sonde a conductance est-il propre au principe de la sonde, ou dépend-il de la vitesse d'écoulement de l'eau ?

«3" Dans l'analogie de Froude, est-il possible de simuler un $\Delta_{0} / 0$ important (par exemple de l'ordre de 0,5 ) en utilisant un $\Delta_{Q} / Q$ différent sur le modele et en compensant cette distorsion par une variation de vitesse?

La concentration $\mathrm{C}_{0}$, répond $\mathrm{M}$. HuFfenus, est de $5 \mathrm{~g} / \mathrm{l}$.

Le temps de réponse de la sonde est de $0,3 \mathrm{~s}$ lorsque la vilesse de l'ecoulement est quasi nulle; elle diminue lorsque cette vitesse croît pour tomber à moins de $0,1 \mathrm{~s}$ dans une veine a grande vitesse.
En ee qui concerne la troisieme question, M. Hurresus precise que la similitude qu'il a utilisée n'est aceptable que si $\Delta_{Q} / Q$ est petit par rapport à 1 ; pour $\Delta_{Q} / Q=0,1$ les resultats sont parfaitement valables; pour des $\Delta_{0} / Q$ de l'ordre de l'unite, il faudrait reprendre le probleme, et introduire sépariment les parametres de similitude $\Delta_{0} / 0$ et Froude.

Dans l'essai de modele de récteur homogène, a-t-on essayé de réaliser une « arrivée multiple » du fluide dans le réacteur, demande-t-on?

M. Hurvexus répond par la négative car, dit-il, divers impératifs de construction et de sécurite interdisaient de compliquer mème légèrement la géométrie du système.

M. Venven pose les deux questions ci-après :

«1" Y a-t-il des effets de convection naturelle à craindre qui modifieraient le champ des vitesses, dans le cour du réacteur à suspension?

«2。 Le réacteur a-t-il été construit et a-t-on pu verifier les résultats obtenus sur la maquette? »

A la première question, M. HuFresus répond que le problème de la convection naturelle a eté traité dans l'une des sessions du Comite 'Technique de la Société Hydrotechnique de France, il y a quelques années (Session des 19 et 20 novembre 1964, Comité Technique n" 76 ); bien que le problème soit complexe, on peut dire qu'il y a, ici, de fortes présomptions pour que la convection naturelle ne joue pas ou tout au moins induise des effets parfaitement négligeables.

M. Hurrexus n'est pas en mesure de répondre à la deuxième question, mais il croit savoir que le réacteur intéressé est actuellement en construction a Arnhem (Pays-Bas).

M. le Président clot la discussion en remerciant le conférencier et en observant que la diffusion moléculaire de la chaleur dans le sodium n'est autre que la conductibilite thermique de ce dernier.

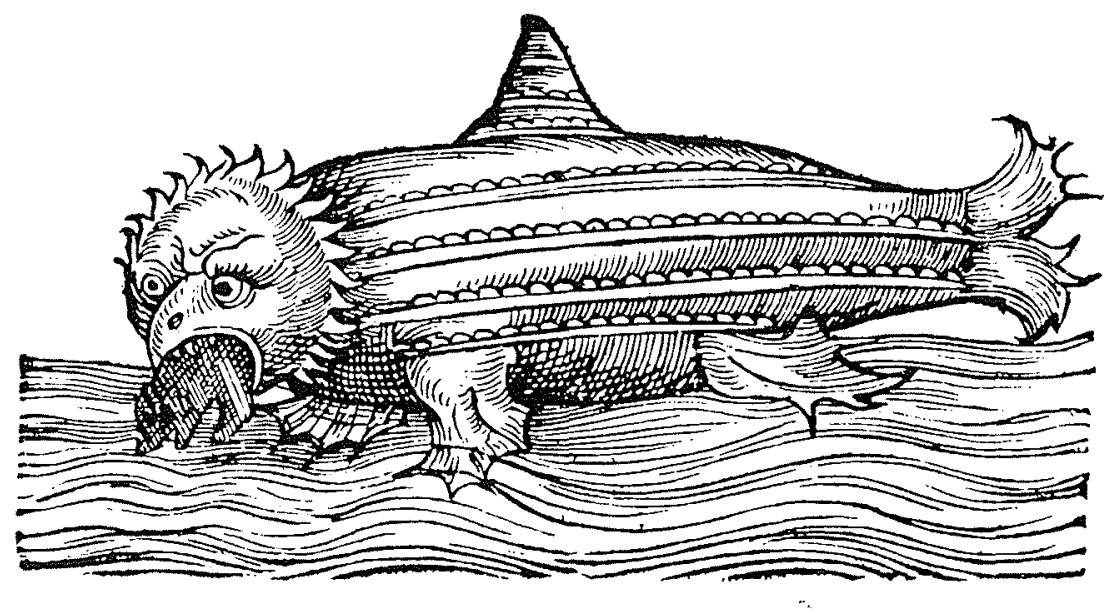

